\title{
Determining the Spectral Resolution of a Charge-Coupled Device (CCD) Raman
} Instrument

\author{
Liu, Chuan; Berg, Rolf W.
}

Published in:

Applied Spectroscopy

Link to article, DOI:

10.1366/11-06508

Publication date:

2012

Document Version

Publisher's PDF, also known as Version of record

Link back to DTU Orbit

Citation (APA):

Liu, C., \& Berg, R. W. (2012). Determining the Spectral Resolution of a Charge-Coupled Device (CCD) Raman Instrument. Applied Spectroscopy, 66(9), 1034-1043. https://doi.org/10.1366/11-06508

\section{General rights}

Copyright and moral rights for the publications made accessible in the public portal are retained by the authors and/or other copyright owners and it is a condition of accessing publications that users recognise and abide by the legal requirements associated with these rights.

- Users may download and print one copy of any publication from the public portal for the purpose of private study or research.

- You may not further distribute the material or use it for any profit-making activity or commercial gain

- You may freely distribute the URL identifying the publication in the public portal

If you believe that this document breaches copyright please contact us providing details, and we will remove access to the work immediately and investigate your claim. 


\title{
Determining the Spectral Resolution of a Charge-Coupled Device (CCD) Raman Instrument
}

\author{
Chuan Liu, Rolf W. Berg* \\ Department of Chemistry, Kemitorvet, Building 207, The Technical University of Denmark (DTU), DK-2800, Kgs. Lyngby, Denmark
}

\begin{abstract}
A new method based on dispersion equations is described to express the spectral resolution of an applied charge-coupled device (CCD) CzernyTurner Raman instrument entirely by means of one equation and principal factors determined by the actual setup. The factors involved are usual quantities such as wavenumber values for the laser and the Raman band, the diffraction grating groove density, the second focal length, the angle between the incident and the diffracted light, and the full width at half-maximum (FWHM) value of the signal on the detector. A basic formula is derived to estimate the spectral resolution of the Raman instrument. An essential feature of the new method is a proposed way to compensate for non-ideality (diffractions, aberrations, etc.) by use of a hyperbola model function to describe the relationship between the width of the entrance slit and the image signal width on the CCD. The model depends on the spectrometer magnification and a diffraction and aberration compensation factor denoted as $A$. $A$ could be approximated as a constant that can be determined by the experimental method. The validity of the new expression has been examined by measuring the band width of the $1332.4 \mathrm{~cm}^{-1}$ diamond Raman fundamental band, excited with two quite different wavelengths (a deep ultraviolet $257.3 \mathrm{~nm}$ laser line and a visible green $514.5 \mathrm{~nm}$ line). A low pressure mercury line at $265.2042 \mathrm{~nm}$ also was applied to give further verification of the given expression. A useful method to find true Raman band widths is also provided. A final finding was that the known significant changes in spectral resolution along the Raman shift axis make static recording and synchronous (extended) scanning modes differ significantly with respect to their resolution properties; this feature has been often overlooked in many contemporary works reporting Raman spectra. A reason for this is that many Raman bands are too wide to show the effect.
\end{abstract}

Index Headings: Spectral resolution; Deep ultraviolet; Raman spectroscopy; Band width; Diamond; Mercury.

\section{INTRODUCTION}

The wavelength of the light sources used for excitation in modern dispersive Raman spectroscopy most often lies in the range from $\sim 800 \mathrm{~nm}$ down to nearly $200 \mathrm{~nm}$. The use of deep ultraviolet (DUV) radiation-in the range below $300 \mathrm{~nm}-$ although used occasionally, is a newly rising technology. DUV Raman spectroscopy has a significant advantage, avoiding the common interference from a high fluorescence background, as discovered a long time ago. ${ }^{1}$ There are, however, also disadvantages to using UV excitation, such as the nontransparency of glassy containers and often the decomposition of the tested samples. ${ }^{2,3}$ Traditional visible light Raman spectroscopy therefore still plays an important role, first of all because it is easier than DUV Raman, and secondly because some samples have interesting resonance Raman spectra in the visible range. ${ }^{4}$

Received 21 October 2011; accepted 8 May 2012.

* Author to whom correspondence should be sent. E-mail: rwb@kemi dtu.dk.

DOI: $10.1366 / 11-06508$
In a flexible Raman research environment it is important to maintain an even and high spectral resolution when switching between instruments and different light sources, changing the setup, or comparing results from different experiments. It is well known that the spectral resolution of a Raman system is affected by many factors, such as grating groove density, entrance slit width, focal length, and so on. In order to estimate and optimize the spectral resolution of an instrument, one must consider all the factors that affect the system resolution. In this paper we attempt to combine all the influencing factors into one single expression. Such an expression has the advantage that it clearly shows the dependence of the spectral resolution on the configurations of the Raman system under different conditions. As will be shown, the expression shows that the spectral resolution exhibits a significant change along the Raman shift axis. This behavior, although known for a long time, is constantly overlooked in many contemporary Raman publications.

With respect to applications in some research areas, knowledge of the precise width of a Raman band is important to characterize the structure of materials. For example the properties and behavior of single-layer graphite (graphene) can be determined by using a Raman technology in which the width and the position of certain characteristic Raman bands may be used as criteria. ${ }^{5}$ In another case, the width of a Raman band could be used to infer the size of germanium nanocrystals. ${ }^{6}$ In such studies one should remember that the spectral resolution resulting from the applied experimental setup directly affects the determined Raman band width. The measurement of a Raman band width should therefore be considered as a convolution relation between the true Raman band and the experimental resolution in the given situation. ${ }^{7}$ Therefore, one should find the true Raman band width by taking the exact spectral resolution of the instrument into consideration. To do this, data for the true experimental resolution must be determined.

The exact spectral resolution of a Raman instrument is typically determined by using a light source with a narrow-line radiation, such as a low pressure mercury lamp or a sample with a well-defined narrow Raman band. ${ }^{8-11}$ In this way the spectral resolution is determined for the particular wavelength(s) or at the corresponding Raman shift(s). Since the spectral resolution is not constant but depends on the wavelength, one needs a well-defined expression to predict (by interpolation or extrapolation) the spectral resolution at positions where the important Raman bands are located, as a prerequisite to finally determine the true Raman band width.

\section{DERIVATION OF THE EXPRESSION}

We will derive the theoretical expression for the spectral resolution of a spectrometer based on the common Czerny- 


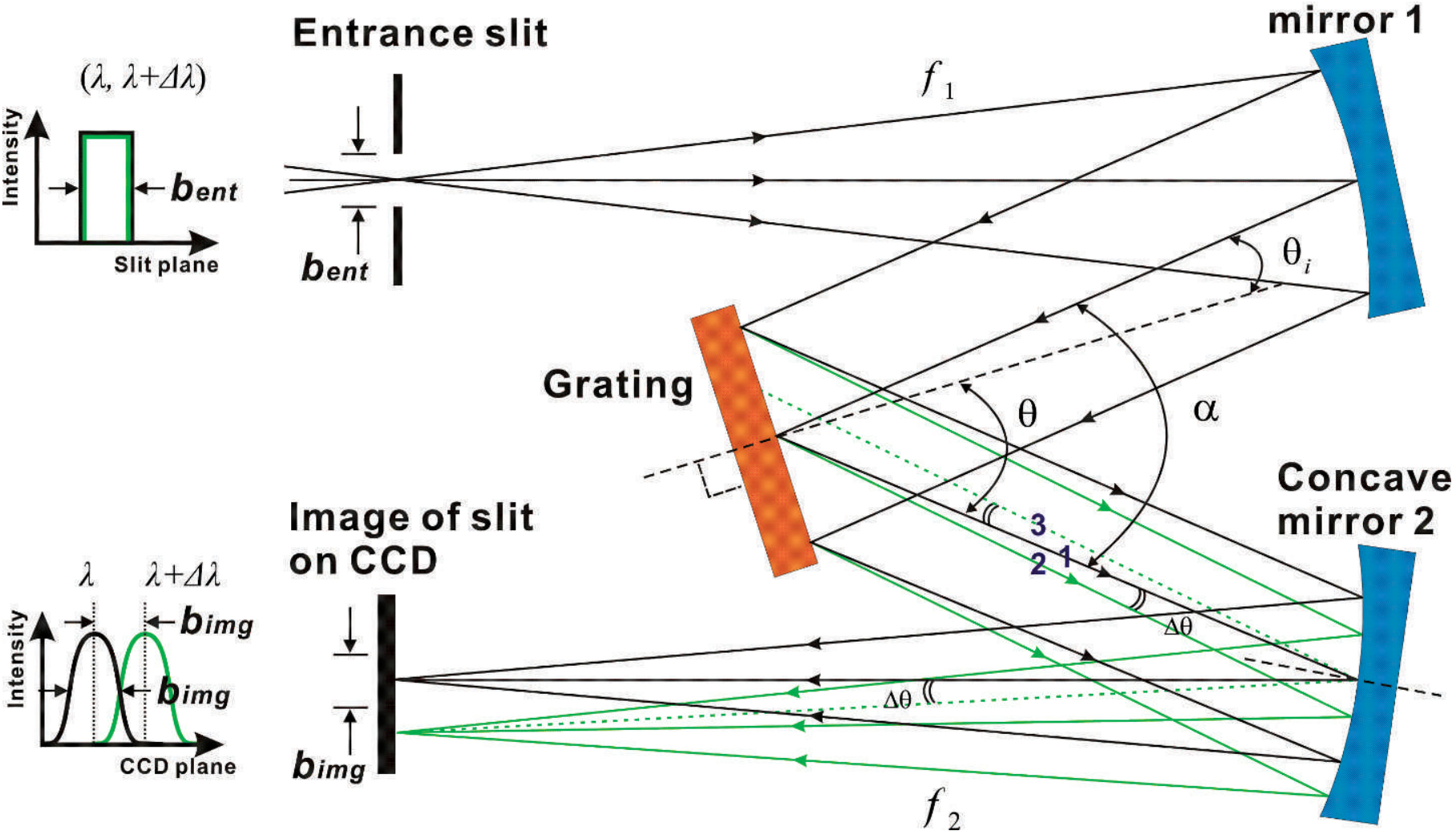

FIG. 1. A schematic of a Czerny-Turner monochromator based spectrometer.

Turner monochromator. ${ }^{12,13}$ The schematics are shown in Fig. 1. Most currently, the spectrometer is equipped with a multichannel charge-coupled device detector (CCD) ${ }^{14}$ replacing previous single-channel detectors behind an exit slit. As we shall see, this complicates the problem. In the following derivation of the expression for the resolution, we are concerned with the central part of the CCD.

The spectral resolution of a Raman system is affected by many factors. We count ten items as shown below (see Table I for a summary of mathematical symbols used):

(1) Exciting laser characterized by the wavelength $\lambda_{\mathrm{L}}$ (or the corresponding wavenumber, $\left.\omega_{\mathrm{L}}\right)$.

(2) Raman wavenumber shift, $\omega_{R}$.

(3) Diffraction grating groove density, $G$.

(4) Angle $\alpha$ between the incident light and the diffracted light.

(5) Focal length, $f$.

(6) Entrance slit width, $b_{\text {ent }}$.

(7) System magnification, $M$.

(8) System diffraction effects of grating and concave mirrors (lenses).

(9) System aberrations.

(10) Pixels of the CCD.

Among these many factors related to the spectral resolution of a Raman instrument, the wavelength dispersing ability $(\Delta \lambda /$ $\Delta \theta$ ) of the grating and the full width at half-maximum (FWHM) of the slit image of a monochromatic light on the CCD are the two key parameters that link up all the factors. Please note that resolution is not affected by the diffraction efficiency of the grating (blazing angle etc.). ${ }^{15-17}$
The wavelength dispersing ability of the grating, here defined as $\Delta \lambda / \Delta \theta$, depends on the spacing of the grating grooves $\left(d\right.$, not shown in Fig. 1) and the incident angle $\left(\theta_{\mathrm{i}}\right)$. The dispersing ability can be derived from the well-known grating relation (Eq. 1) as shown below. $\theta$ is the diffractive angle, i.e., the angle between the surface normal and the diffracted beam, and the order of diffraction is set to one (normally the first order is used in Czerny-Turner monochromators to get the highest throughput):

$$
d \cdot\left[\sin \left(\theta_{i}\right)+\sin (\theta)\right]=\lambda
$$

By differentiation one gets the relation

$$
\frac{\partial \lambda}{\partial \theta}=d \cdot \cos (\theta) \Rightarrow \frac{\Delta \lambda}{\Delta \theta}=d \cdot \cos (\theta)
$$

Assuming that monochromatic light, say of wavelength $\lambda$, is entering through the entrance slit, then an image of the entrance slit (with the physical width $b_{\text {ent }}$ and a color corresponding to $\lambda$ ) will be formed on the detector (CCD), by means of the concave mirrors (see Fig. 1). Because of diffractions and aberrations, the intensity profile of the image is no longer a rectangle curve but will be a bell-shaped curve, corresponding to the signal obtained with a CCD having a sufficiently narrow pixel size (such that at least a few pixels are sensing the curve ${ }^{11,16,17}$ ). The FWHM of the bell-shaped curve is denoted as $b_{\text {img }}$. Furthermore, to analyze the situation more carefully, the slit image (or signal versus wavelength) due to the angular dispersion of the grating will be depicted differently by, e.g., two different wavelengths, say $\lambda$ and $(\lambda+\Delta \lambda)$ (see Fig. 1). 
When the two images corresponding to different grating diffraction angles, $\theta$ and $(\theta+\Delta \theta)$, are juxtaposed at a distance corresponding to the FWHM, then these two peaks can be considered to be just distinguishable (or resolved). This situation characterizes the resolving power $\Delta \lambda$ of the spectrograph. The opening angle $\Delta \theta$ at the grating (between the rays $\mathbf{1}$ and $\mathbf{2}$ in Fig. 1) can be seen (by geometry, because ray $\mathbf{2}$ and $\mathbf{3}$ are parallel) to form at the Concave mirror 2 another angle $\Delta \theta$ of equal magnitude, because rays $\mathbf{1}$ and $\mathbf{3}$ are reflected in the same point. From this and the near right angle hitting of the image on the slit, it therefore follows that $b_{\text {img }}$, $\Delta \theta$, and the second focal length $f_{2}$ are related in the following way:

$$
\Delta \theta=\frac{b_{\mathrm{img}}}{f_{2}}
$$

The $\Delta \theta$ angle is equal to the grating dispersive angle for these two just distinguishable wavelengths, as seen in Fig. 1.

By substitution of Eq. 3 into Eq. 2, we obtain the spatial resolution as

$$
\Delta \lambda=\frac{b_{\mathrm{img}}}{f_{2}} \cdot d \cdot \cos (\theta)
$$

To eliminate $\theta$ from this expression we introduce the angle $\alpha$ between the direction of the incident light and the diffracted light, as shown in Fig. 1 (be careful with the sign convention of the angles $\theta$ and $\theta_{i}$; they are measured clockwise from the grating normal).

$$
\alpha=\theta-\theta_{i}
$$

This angle $\alpha$ is normally kept constant for well-designed instruments, the condition for this constancy being that the grating rotation is done around an axis chosen to be parallel with the grating surface and centrally placed. Then we rearrange Eq. 1 from a sum to a product by use of a standard trigonometric formula:

$$
\begin{array}{r}
d \cdot\left[\sin \left(\theta_{i}\right)+\sin (\theta)\right]=\lambda \Rightarrow \\
d \cdot\left[2 \cdot \sin \left(\frac{\theta+\theta_{i}}{2}\right) \cdot \cos \left(\frac{\theta-\theta_{i}}{2}\right)\right]=\lambda
\end{array}
$$

By substituting Eq. 5 into Eq. 6, we get the equation:

$$
d \cdot\left[2 \cdot \sin \left(\frac{2 \theta-\alpha}{2}\right) \cdot \cos \left(\frac{\alpha}{2}\right)\right]=\lambda
$$

Then, solving for $\theta$ we have

$$
\theta=\arcsin \left[\frac{\lambda}{2 \cdot d \cdot \cos \left(\frac{\alpha}{2}\right)}\right]+\frac{\alpha}{2}
$$

\begin{tabular}{|c|c|}
\hline A & The diffractions and aberrations compensation factor (DACF) \\
\hline$b_{\mathrm{ent}}$ & Width of entrance slit \\
\hline$b_{\text {img }}$ & FWHM of the entrance slit image for $M=1$ \\
\hline$b_{\text {limit }}$ & The limitation FWHM of the slit image for $M=1$ \\
\hline$D A C F$ & The diffractions and aberrations compensation factor \\
\hline$D$ & The spacing of the grating grooves \\
\hline FWHM & Full width at half-maximum \\
\hline$f_{1}$ & Focal length of concave mirror 1 or lens 1 \\
\hline$f_{2}$ & Focal length of concave mirror 2 or lens 2 \\
\hline$G$ & Diffraction grating groove density \\
\hline$g_{\mathrm{L}}(\omega)$ & Spectrum of the exciting laser \\
\hline$g_{\mathrm{LO}}$ & $\begin{array}{l}\text { Normalizing constant corresponding to maximum of the laser } \\
\text { peak }\end{array}$ \\
\hline$g_{\mathrm{LR}}(\omega)$ & Profile of the Raman band excited by a laser \\
\hline$g_{\mathrm{M}}(\omega)$ & Profile of the measured Raman band \\
\hline$g_{\mathrm{R}}\left(\omega_{\mathrm{r}}\right)$ & Profile of a true Raman band \\
\hline$g_{\mathrm{R} 0}$ & Constant corresponding to maximum of the true Raman peak \\
\hline$g_{\mathrm{S}}\left(\omega_{\mathrm{S}}\right)$ & Signal obtained at the detector for a monochromatic line \\
\hline$g_{\text {So }}$ & $\begin{array}{l}\text { Normalizing constant for the spectral contribution (expanded by } \\
\text { spectrometer) of a monochromatic light }\end{array}$ \\
\hline$M$ & System magnification \\
\hline$\alpha$ & Angle between the incident light and the diffracted light \\
\hline$\Delta \lambda$ & Difference between two close wavelengths \\
\hline$\lambda_{L}$ & Wavelength of the exciting laser \\
\hline$\Delta \omega$ & Theoretical spectral resolution of spectrometer $\left[\mathrm{cm}^{-1}\right]$ \\
\hline$\omega_{0}$ & $\begin{array}{l}\text { Central Raman wavenumber shift for static grating measurement } \\
{\left[\mathrm{cm}^{-1}\right]}\end{array}$ \\
\hline$\omega_{1}$ & $\begin{array}{l}\text { Narrow incremental section (a delta function) of the laser } \\
\text { spectrum }\left[\mathrm{cm}^{-1}\right]\end{array}$ \\
\hline$\omega_{2}$ & Wavenumber of integration in convolution $\left[\mathrm{cm}^{-1}\right]$ \\
\hline$\omega_{\mathrm{L}}$ & Absolute wavenumber of the exciting laser $\left[\mathrm{cm}^{-1}\right]$ \\
\hline$\Delta \omega_{\mathrm{L}}$ & Linewidth of the exciting laser $\left[\mathrm{cm}^{-1}\right]$ \\
\hline$\Delta \omega_{\mathrm{M}}$ & Measured bandwidth of Raman band $\left[\mathrm{cm}^{-1}\right]$ \\
\hline$\omega_{\mathrm{r}}$ & The variable of Raman shift $\left[\mathrm{cm}^{-1}\right]$ \\
\hline$\omega_{\mathrm{R}}$ & Raman wavenumber shift $\left[\mathrm{cm}^{-1}\right]$ \\
\hline$\Delta \omega_{\mathrm{R}}$ & True Raman bandwidth $\left[\mathrm{cm}^{-1}\right]$ \\
\hline$\omega_{\mathrm{s}}$ & $\begin{array}{l}\text { The variable of the wavenumber difference between the real } \\
\text { Raman signal and the measured Raman signal }\left[\mathrm{cm}^{-1}\right]\end{array}$ \\
\hline$\Delta \omega_{\mathrm{S}}$ & Experimental spectral resolution of spectrometer $\left[\mathrm{cm}^{-1}\right]$ \\
\hline$\theta$ & Grating diffraction angle \\
\hline$\Delta \theta$ & Difference between two close diffraction angles \\
\hline$\theta_{\mathrm{i}}$ & Grating incident angle \\
\hline
\end{tabular}

By substituting Eq. 8 into Eq. 4 we get the expression

$$
\Delta \lambda=\frac{b_{\mathrm{img}}}{f_{2}} \cdot d \cdot \cos \left\{\arcsin \left[\frac{\lambda}{2 \cdot d \cdot \cos \left(\frac{\alpha}{2}\right)}\right]+\frac{\alpha}{2}\right\}
$$

TABLE I. Mathematical symbols.

Normally, Raman band absolute positions are expressed as $\omega$ in wavenumber units. In order to derive a practical expression in wavenumber units, we apply these conversions:

$$
\omega=\frac{1}{\lambda} \Rightarrow \frac{\partial \omega}{\partial \lambda}=-\frac{1}{\lambda^{2}}
$$

Hence, for small absolute values of frequency difference, $\Delta \omega$, one gets the approximation

$$
\Delta \omega=\omega^{2} \cdot \Delta \lambda=\left(\omega_{L}-\omega_{R}\right)^{2} \cdot \Delta \lambda
$$

where $\omega$ is the Raman scattering absolute wavenumber position, $\omega_{\mathrm{L}}$ is the laser line absolute wavenumber value, and $\omega_{\mathrm{R}}$ is the Stokes wavenumber shift of the scattered light.

Instead of specifying the separation between the grating grooves $(d$, not shown in Fig. 1), one often uses the density of the grating grooves (i.e., the number $G$ of grooves per unit length) to classify the grating:

$$
d=\frac{1}{G}
$$

After substitution and unit conversion, the spectral resolution function becomes 


$$
\begin{aligned}
\Delta \omega & {\left[\omega_{\mathrm{L}}, \omega_{\mathrm{R}}, G, f_{2}, \alpha, b_{\mathrm{img}}\right] } \\
= & \left(\omega_{\mathrm{L}}-\omega_{\mathrm{R}}\right)^{2} \\
& \quad \frac{b_{\mathrm{img}}}{G \cdot f_{2}} \cdot \cos \left\{\arcsin \left[\frac{G}{2 \cdot\left(\omega_{\mathrm{L}}-\omega_{\mathrm{R}}\right) \cdot \cos \left(\frac{\alpha}{2}\right)}\right]+\frac{\alpha}{2}\right\}
\end{aligned}
$$

It should be noted, because the unit of wavenumber is usually $\mathrm{cm}^{-1}$, when Eq. 12 is applied for numerical calculations, the units of length for all the parameters must be transformed to $\mathrm{cm}$.

In the above expression, there are six variables; five of these variables correspond to five of the factors (items 1-5) that were mentioned in the beginning. Items 6-9 must also have influence on the relationship between the widths of the entrance slit $\left(b_{\text {ent }}\right)$ and the image thereof $\left(b_{\text {img }}\right)$, thereby affecting the spectral resolution. For monochromatic light, the limiting FWHM of the peak of the image is governed by the diffraction effects and the aberrations (items 8 and 9). However, this is not easily expressed and we therefore seek an empirical relationship between $b_{\text {img }}$ and $b_{\text {ent }}$. Hence, considering the experimental results as shown later, it seems that the spectral resolution $(\Delta \omega)$ versus the width of the entrance slit $\left(b_{\text {ent }}\right)$ looks like a hyperbolic curve. Furthermore, $\Delta \omega$ and $b_{\text {img }}$ have a direct proportional relationship. Therefore, we propose using a hyperbola function $\left(b_{\text {ent }}\right.$ versus $\left.b_{\text {img }}\right)$ as a model for the relationship between $b_{\text {ent }}$ and $b_{\text {img. If so, we }}$ would have a relationship between $b_{\mathrm{img}}$ and $b_{\mathrm{ent}}$ of the kind:

$$
b_{\text {img }}=M \cdot \sqrt{\left(b_{\text {ent }}\right)^{2}+\left(b_{\text {limit }}\right)^{2}}
$$

where

$$
M=\frac{f_{2}}{f_{1}}
$$

In this expression, $M$ is the system magnification, defined by the focal length $f_{2}$ of the second concave mirror, divided by the focal length $f_{1}$ of the first concave mirror (see Fig. 1). $b_{\text {limit }}$ can be defined as the smallest possible limitation FWHM of the slit image when $M=1$. The limitation is caused by the system diffractions and the aberrations. In practice the system diffractions play a more important role than the aberrations. According to the diffraction equation of a grating and a circular aperture, ${ }^{15}$ a reasonably approximate expression of $b_{\text {limit }}$ would be

$$
b_{\text {limit }}=A \cdot \lambda=\frac{A}{\left(\omega_{\mathrm{L}}-\omega_{\mathrm{R}}\right)}
$$

where $A$ could be approximated as a constant for a spectrometer with a certain setup. Considering that $A$ depends on the system diffractions and aberrations, we denote $A$ as the diffractions and aberrations compensation factor (DACF) of the used spectrometer system.

To summarize the results so far: In the above equations 12 and 13, the values of all the factors except $A$ can easily be determined for a particular Raman instrumental situation.

When the width of the entrance slit $b_{\text {ent }}$ is broad enough (such as: $>100 \mu \mathrm{m}$ at $500 \mathrm{~nm}$ ), the effects of diffraction and aberration become weak, and therefore we may do the approximation:

$$
b_{\mathrm{img}} \approx M \cdot b_{\mathrm{ent}} \quad\left(b_{\mathrm{ent}}>100 \mu \mathrm{m}\right)
$$

This can become the experimental method to verify whether Eq. 12 gives a correct description. In addition, we can use a set of measurements to determine $A$ and check whether the hyperbola approximation, Eq. 13, is indeed a good model.

Concerning Eq. 12, it is worth noting that the CCD pixel width size, for normally adopted Raman instrument layouts, is narrower than the width of common Raman bands. Thus, the $\mathrm{CCD}$ records the spectrum band as a digital profile from several pixels. The digital profile of the spectrum band will constitute a not very smooth curve, at least when the widths of the CCD pixels and the spectrum band are comparable (see later). The FWHM of a not very smooth curve has a deviation from the true FWHM of the spectrum band, hence affecting the spectral resolution of a spectrometer. This is one reason why the theoretical curves - as we shall see-do not fit well with the experimental curves in the range of narrowly opened slits $\left(b_{\text {ent }}\right.$ $<20 \mu \mathrm{m})$.

In practice the spectrum band is broadened not only by the limited resolution of the spectrometer but also by the line width of the exciting laser. In general the FWHM of the spectrum band will be determined by the convolution of these two causes of broadening. Assuming a Gaussian line profile, an approximation of the FWHM can be provided by this relationship (see Appendix 1):

$$
\Delta \omega_{\mathrm{M}}=\sqrt{\left(\Delta \omega_{\mathrm{R}}\right)^{2}+\left(\Delta \omega_{\mathrm{S}}\right)^{2}+\left(\Delta \omega_{\mathrm{L}}\right)^{2}}
$$

Here $\Delta \omega_{M}$ is the measured spectrum bandwidth, $\Delta \omega_{R}$ is the true Raman spectrum bandwidth, $\Delta \omega_{S}$ is the spectral resolution of the spectrometer, and $\Delta \omega_{\mathrm{L}}$ is the linewidth of the excited laser.

This equation (Eq. 16) is an interesting result. As the laser line width, $\Delta \omega_{\mathrm{L}}$, is typically known (e.g., given by manufacturer), and $\Delta \omega_{M}$ is the experimental value, we can calculate the spectral resolution $\Delta \omega_{\mathrm{S}}$ of the spectrometer if the true Raman bandwidth, $\Delta \omega_{\mathrm{R}}$, is also known. On one hand, this is an experimental method to find the spectral resolution of the spectrometer at a certain absolute wavenumber. To do this the spectral resolution compensation factor $A$, or the $D A C F$ parameter, can be determined by Eqs. 12, 13, and 14, and then the spectral resolution of the spectrometer in the whole wavenumber range can be determined. On the other hand, we can use Eq. 16 to determine the true Raman bandwidth for the testing sample, as soon as the spectral resolution is totally known.

\section{EXPERIMENTS}

Samples. A monocrystalline natural diamond slab with a thickness of $2 \mathrm{~mm}$ that did not emit fluorescence was used. Diamond has a characteristic strong Raman band, located at $1332.4 \mathrm{~cm}^{-1}$, that acted as a suitable narrow line source. The band width of the diamond line is known to be $\Delta \omega_{\mathrm{R}}=1.2$ $\mathrm{cm}^{-1} \cdot{ }^{18}$ Another useful source was a low pressure $\mathrm{Hg}(\mathrm{Ar})$ pencil style calibration lamp from Oriel Instruments.

Instrumentation. As exciting light source we used a Lexel 95-SHG-QS Argon gas ion laser (from Cambridge Laser Laboratories, Inc.) working in direct or second harmonic generation (SHG) mode. The wavelengths (powers) applied 
TABLE II. Experimental details.

\begin{tabular}{ccccc}
\hline Exciting wavelength $(\mathrm{nm})$ & Grating groove density $G\left(\mathrm{~mm}^{-1}\right)$ & Diffraction angle $\alpha(\mathrm{deg})$ & Focal length $f_{1}(\mathrm{~mm})$ & Focal length $f_{2}(\mathrm{~mm})$ \\
\hline 514.5 & 2400 & 37 & 140 & 250 \\
257.3 & 3600 & 37 & 155 & 250 \\
\hline
\end{tabular}

TABLE III. Comparison of the experimental and theoretical results for the spectral resolution for a wide entrance slit, $b_{\mathrm{ent}}=140 \mu \mathrm{m}$.

\begin{tabular}{cccc}
\hline Exciting wavelength $\lambda(\mathrm{nm})$ & $\begin{array}{c}\text { The measurements of Raman } \\
\text { bandwidth } \Delta \omega_{\mathrm{M}}\left(\mathrm{cm}^{-1}\right)\end{array}$ & $\begin{array}{c}\text { Experimental results of spectral } \\
\text { resolution } \Delta \omega_{\mathrm{S}}\left(\mathrm{cm}^{-1}\right)\end{array}$ & $\begin{array}{c}\text { Theoretical results of spectral } \\
\text { resolution } \Delta \omega\left(\mathrm{cm}^{-1}\right)\end{array}$ \\
\hline 514.5 & 6.77 & 6.66 & 6.23 \\
257.3 & 23.80 & 23.77 & 23.24 \\
\hline
\end{tabular}

were $514.5 \mathrm{~nm}(10 \mathrm{~mW})$ and $257.3 \mathrm{~nm}(1 \mathrm{~mW})$. The linewidth $\Delta \omega_{\mathrm{L}}$ for the $514.5 \mathrm{~nm}$ line was $0.17 \mathrm{~cm}^{-1}$. Since the $257.3 \mathrm{~nm}$ line is generated from the $514.5 \mathrm{~nm}$ line, the width should be narrower but we have also taken the value $0.17 \mathrm{~cm}^{-1}$ as an approximation for the $257.3 \mathrm{~nm}$ line width.

A flexible InVia Reflex Raman spectrometer system with a $\times 20$ objective from Renishaw, plc. was used to record the spectra. The optical principle of the InVia Reflex spectrometer is equivalent to a single classical Czerny-Turner monochromator. Instead of concave mirrors, mirrors combined with lenses are used in the Renishaw system. Compared to the concave mirrors, lenses have the well-known problem of chromatic aberrations, and even for achromatic lenses it is not possible to cover the whole range from UV to the visible; therefore, the lenses must (like the grating) be exchanged in order to suit the whole range of wavelengths from UV to visible. The details of the experiments are shown in Table II.

Examination of the Expression of Spectral Resolution, Eq. 12. As noted above, when the width of the entrance slit $\left(b_{\text {ent }}\right)$ is broad enough, the effects of diffraction and aberrations can be ignored, and therefore the spectral resolution can be calculated theoretically by means of Eq. 12. After that, one can compare with the experimental results to examine whether Eq. 12 is correct. We take $b_{\text {ent }}=140 \mu \mathrm{m}$ as the condition for a broad slit-width experiment. For this slit width, measurements of the Raman band widths of diamond are shown in Table III. After applying the values of $\Delta \omega_{M}$ to Eq. 16, the experimental results found for the spectral resolution $\Delta \omega_{S}$ are shown in Table III. The theoretical results of the spectral resolution $\Delta \omega$ are also shown, calculated by Eqs. 12 and 13 .

As shown in Table III, there is good correspondence between the experimental results $\left(\Delta \omega_{\mathrm{S}}\right)$ and the theoretical results $(\Delta \omega)$; only a few percent differences are seen. This is to be expected because we ignored the effect of the aberrations, as previously noted.

Hyperbola Model for the Spectral Resolution. According to Eq. 13, when the width $\left(b_{\text {ent }}\right)$ of the entrance slit decreases, the effect of diffraction and aberrations $\left(b_{\text {limit }}\right)$ becomes important. Therefore, these effects cannot be ignored for narrow entrance slits. To investigate the situation, the Raman band widths $\left(\Delta \omega_{M}\right)$ of a diamond sample were measured for various entrance slit widths $\left(b_{\text {ent }}\right)$, from $10 \mu \mathrm{m}$ to $180 \mu \mathrm{m}$. The obtained results are shown in Fig. 2. In order to compare the results in a clear way, the spectra were auto-scaled to have the same maximum (set to unity). To explain the shape of the curves in Fig. 2 we realize that the true band width of the diamond Raman fundamental is quite narrow $\left(1.2 \mathrm{~cm}^{-1}\right)$. If a strictly monochromatic light source illuminates a wide entrance slit and is detected on an ideal CCD, then the detected band will have a trapezoidal shape. This explains why we are seeing more or less flat-topped spectra in Fig. 2.

The analyses of these results are given in Fig. 3. Here, the large green points represent the measurements of the Raman band width (achieved by measuring FWHM without modeling) versus the slit width. From these results we can calculate the experimental spectral resolution (the red small points) by using Eq. 16.

If one chooses an appropriate number for the spectral resolution compensation factor $A$ and substitutes it into Eq. 14, then one can plot an estimate of the spectral resolution using Eq. 12. The chosen number for $A$ can be varied by iteration to find better or worse fits to the experimental red resolution
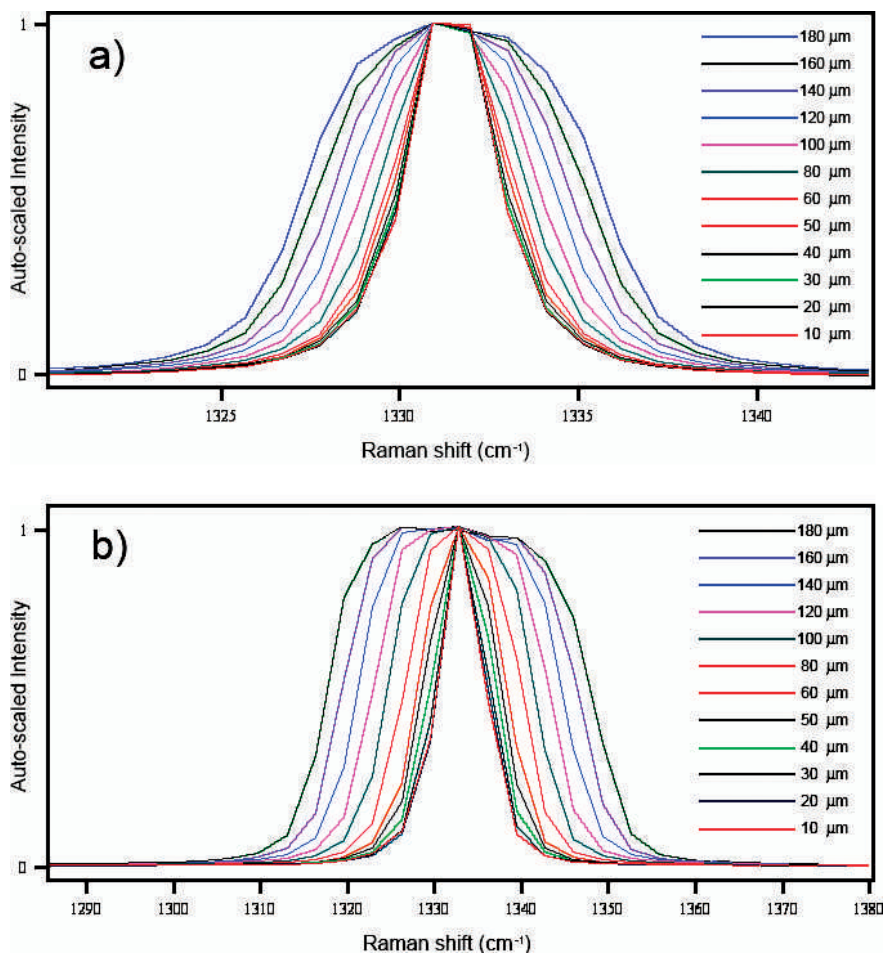

FIG. 2. Auto-scaled Raman spectra of the $1332.4 \mathrm{~cm}^{-1}$ band of a diamond obtained by use of various slit widths (given in $\mu \mathrm{m}$ ), and (a) excited with 514.5 $\mathrm{nm}$ radiation and (b) with $257.3 \mathrm{~nm}$. 


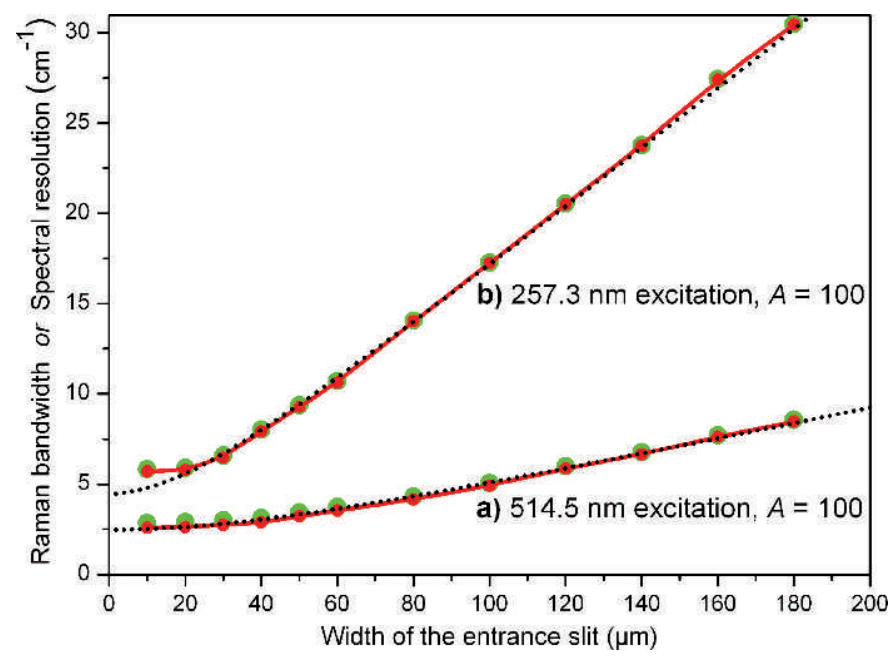

FIG. 3. The wide green points are the measurements of Raman bandwidth $\Delta \omega_{M}$ of diamond for various slit widths. The narrow red points, connected by red curves, are the experimental spectral resolution data $\Delta \omega$ obtained by means of Eq. 16. The black dotted curve shows the spectral resolution modeling results (theoretical spectral resolution) based on Eqs. 12, 13, and 14. (a) The wavelength of the exciting light source was $514.5 \mathrm{~nm}$ and $A$ was set to 100 . (b) The wavelength of the exciting light source was $257.3 \mathrm{~nm}$ and $A$ was set to 100 .

points in Fig. 3. When $A=100$, we obtained the shown approximate model curves (black dotted curves) in Fig. 3.

The experimental results (red curves) were fitted well by the theoretical results (black dotted curves) except for narrow slit openings $(<20 \mu \mathrm{m})$. One reason is the effect of the CCD spectral resolution. Other reasons are that the effects of diffraction and aberrations become more important. As obvious from Fig. 2 the un-smoothed faceted spectrum profiles must be caused by the width of the CCD pixels. One might be tempted from Fig. 2 to use narrow slits. However, slits as narrow as $<20 \mu \mathrm{m}$ are not practical for real measurements because the Raman signals are extremely decreased when such narrow slits are used (the weakness of the narrow-slit spectra is not obvious from the spectra after setting the auto-scaled intensity to unity).

\section{HOW TO CHOOSE A SUITABLE WIDTH OF THE ENTRANCE SLIT}

For a practical Raman spectrum measurement, in order to choose a suitable width of the entrance slit $b_{\text {ent }}$, one needs to consider both the signal intensity and the spectral resolution. Then, the limitation FWHM of the slit image $b_{\text {limit }}$ could be used as an important reference value. According to equations Eqs. 13 and 14, when

$$
b_{\mathrm{ent}}=b_{\mathrm{limit}}=A \cdot \lambda
$$

we can calculate

$$
b_{\text {img }}=\sqrt{2} \cdot M \cdot b_{\text {limit }}
$$

This means that the achieved spectral resolution will be $\sqrt{2}$ times the limitation value.

Using the obtained value of $A$ for the visible laser line 514.5 nm, we get

$$
b_{\text {limit }}=A \cdot \lambda=100 \cdot 514.5 \mathrm{~nm}=51.45 \mu \mathrm{m}
$$

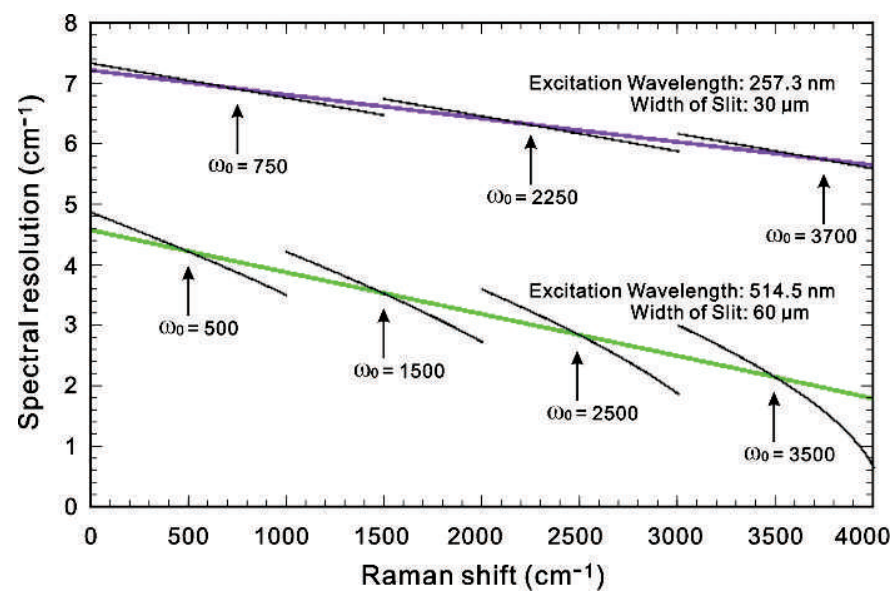

FIG. 4. The observed dependence of the spectral resolution along the Raman shift axis. The green and violet lines show the theoretical results calculated by use of Eq. 12 for only the central part of the CCD (in extended scanning mode using only a few pixels). The black lines show the spectral resolution increments in four spectrograms and three spectrograms, for $514.5 \mathrm{~nm}$ and $257.3 \mathrm{~nm}$ laser excitation, respectively, obtained in static mode recordings, using many pixels and settings of the central pixel of the CCD at Raman shift wavenumbers of $\omega_{0}=500,1500,2500$, and $3500 \mathrm{~cm}^{-1}$ for $514.5 \mathrm{~nm}$ excitation, and $\omega_{0}=750,2250$, and $3750 \mathrm{~cm}^{-1}$ for $257.3 \mathrm{~nm}$ excitation, respectively.

and for the DUV laser line $257.3 \mathrm{~nm}$,

$$
b_{\text {limit }}=A \cdot \lambda=100 \cdot 257.3 \mathrm{~nm}=25.73 \mu \mathrm{m}
$$

For the case of Stokes scattering, the wavelength of the Raman signal is longer than the wavelength of the laser line. Therefore, as a compromise choice, slit widths of about $60 \mu \mathrm{m}$ for 514.5 $\mathrm{nm}$ and about $30 \mu \mathrm{m}$ for $257.3 \mathrm{~nm}$ are reasonably adopted for practical measurements.

\section{THE DEPENDENCE OF THE SPECTRAL RESOLUTION ALONG THE RAMAN SHIFT AXIS}

According to Eq. 12, it is possible to plot the theoretical spectral resolution in the range of Raman shifts from 0 to 4000 $\mathrm{cm}^{-1}$, as shown in Fig. 4 (green and violet line). Note that the resolution changes significantly along the abscissa. Since Eq. 12 predicts the spectral resolution for the central part of the $\mathrm{CCD}$, it does not describe the situation for the spectral resolution in an exact way in the Raman spectrum recorded by using the whole CCD. In the non-central part of the CCD, the case does not strictly satisfy Eq. 5, $\alpha=\theta-\theta_{\mathrm{i}}$, but rather an approximate version, $\alpha \approx \theta-\theta_{\mathrm{i}}$. Actually, for a static grating measurement, $\theta_{i}$ is a constant (see Fig. 1); therefore, an expression of the spectral resolution, Eq. 12, can be obtained by using $\theta_{\mathrm{i}}$ instead of $\alpha$ (see Appendix 2). The black lines in Fig. 4 show the variation of the theoretical spectral resolution for a series of static measured multi-channel spectrograms (static as opposed to so-called extended scanning, a feature in the Renishaw spectrometer implemented by moving the grating and the charge detected on the CCD camera synchronously ${ }^{19-23}$ ).

Experiments with Mercury Lines. The low pressure mercury lamp emits several narrow lines in the DUV range. Therefore, it can be used as a wavelength calibration light source for DUV Raman instruments, and furthermore it is well suited for determination of the spectral resolution in the DUV range. ${ }^{11}$ Particularly, there are three close lines with wave- 


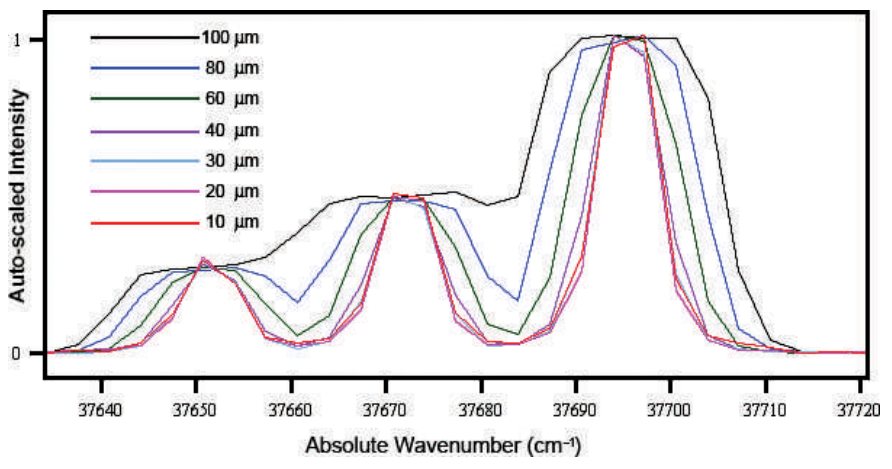

FIG. 5. Auto-scaled intensity of mercury lines at $37695.57,37672.29$, and $37651.86 \mathrm{~cm}^{-1}$, obtained by the use of various slit widths (given in $\mu \mathrm{m}$ ).

lengths of 265.2042, 265.3681, and $265.5121 \mathrm{~nm}$ in air, or $265.2831,265.4471$, and $265.5911 \mathrm{~nm}$ in vacuum $^{24}(\sim 0.01$ $\mathrm{nm}$ larger values have been given in Ref. 25). These narrow lines correspond to absolute wavenumbers of 37695.57 , 37672.29 , and $37651.86 \mathrm{~cm}^{-1}$, respectively, and they provide a direct view of the resolution capability of DUV Raman instruments.

These three lines of the low pressure mercury lamp were measured using a spectrometer setup similar to the one used for recording the Raman band of diamond excited with the 257.3 $\mathrm{nm}$ laser. The grating was set to a CCD central value of $265.2042 \mathrm{~nm}$ (the line of $37695.57 \mathrm{~cm}^{-1}$ ) and various openings of the entrance slit were applied, as shown in Fig. 5.

From these spectra we determined the FWHM values of the lines by means of software in the Renishaw WiRE ${ }^{\mathrm{TM}} 3.0$ package. The principle and the results are shown in Fig. 6. Note that a suitable curve resolution algorithm is indispensable during the evaluation of the FWHM values of a spectrum band because neighboring lines are obviously affecting each other when the lines are close enough, as seen in Fig. 6.

When all the curves in Fig. 5 have been curve resolved as for the example in Fig. 6, a data set is obtained that forms the basis for Fig. 7. Here, wide green points depict a plot of the line widths for the $265.2042 \mathrm{~nm}$ mercury line versus the various slit openings.

From these line widths $\left(\Delta \omega_{M}\right.$, the wide green points in Fig.

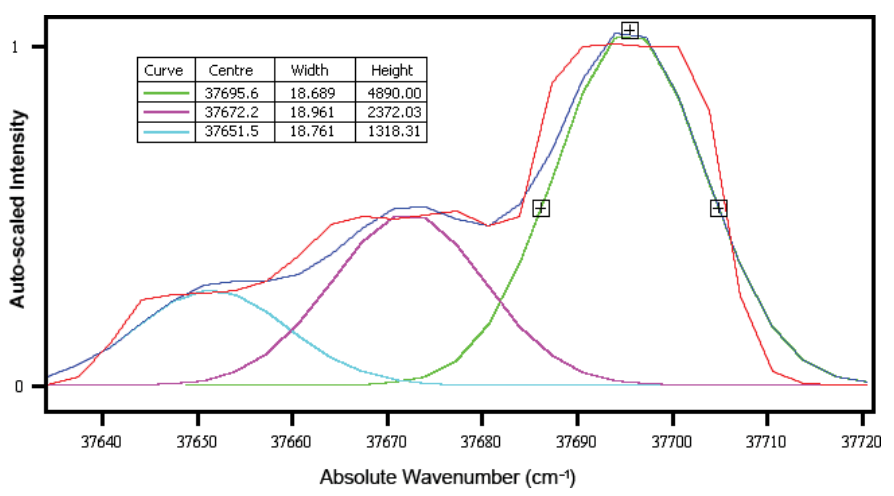

FIG. 6. Example showing how to find the FWHM of the mercury line for the spectrum recorded for a slit width of $100 \mu \mathrm{m}$ (red curve). The FWHMs of the spectrum bands (main peaks of the curve) contain the contributions of the neighboring lines. The green, pink, and light blue curves and their blue sum are the Gaussian simulations of these three bands. The maximum and FWHM of the mercury line at $37695.57 \mathrm{~cm}^{-1}$ are indicated with $\boxplus$ signs (note that the heights are not the auto-scaled but the predicted intensities of the model).

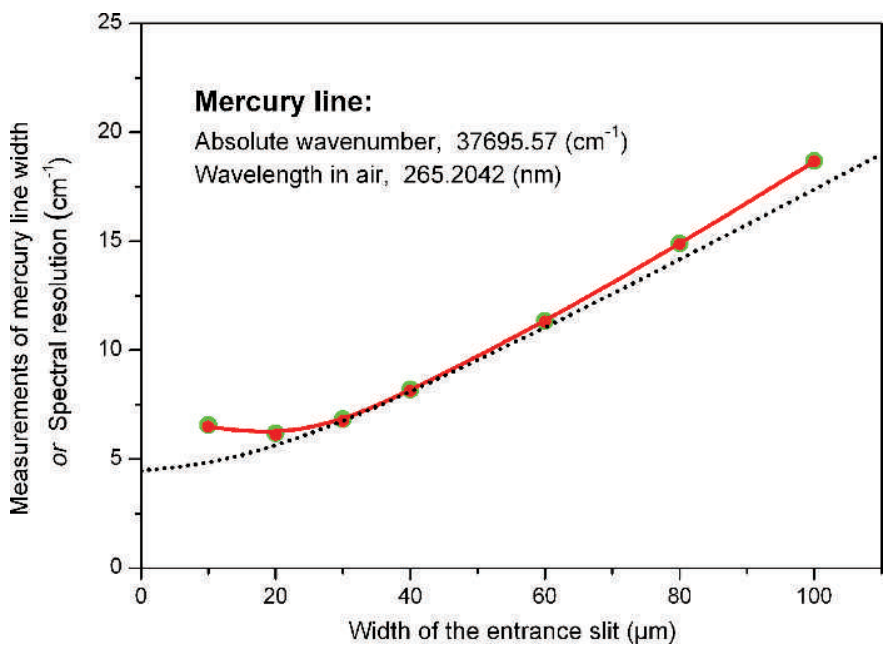

FIG. 7. The wide green points are the measurements of the linewidth $\Delta \omega_{M}$ of the low pressure mercury lamp for various slit widths. The narrow red points, connected by the red curve, are the experimental spectral resolution data $\Delta \omega$, obtained by means of Eq. 16. The black dotted curve is the spectral resolution modeling results (theoretical spectral resolution) based on Eqs. 12, 13, and 14 $A=100$ was determined during the diamond experiments.

7) the corresponding experimental spectral resolutions of the spectrometer $\left(\Delta \omega_{S}\right.$, the narrow red points in Fig. 7) are obtained, again as in Fig. 3 by means of Eq. 16, but now with $\Delta \omega_{\mathrm{L}}=0$ and $\Delta \omega_{\mathrm{R}} \approx 1 \mathrm{~cm}^{-1}$. Here $\Delta \omega_{\mathrm{R}}$ is the natural (or true) line width of the mercury line. We consider it to be less than 1 $\mathrm{cm}^{-1}$ but the approximation of 1 suffices because it is not an important factor when the spectral resolution is much bigger than $1 \mathrm{~cm}^{-1}$.

The experimental spectral resolution curve of the spectrometer (red curve) — determined by this curve resolution technique on the $265.2042 \mathrm{~nm}$ mercury line data-compares well with the theoretical spectral resolution results (black dotted curve) obtained by using Eqs. 12, 13, and 14 with $A=100$, which was determined from diamond experiments as shown in Fig. 7.

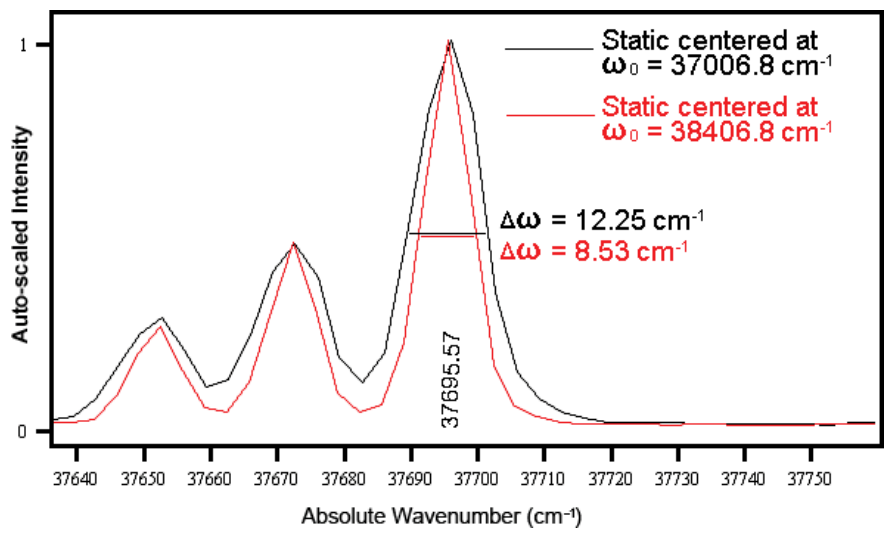

FIG. 8. Example of spectra obtained for mercury lines at $37695.57,37672.29$ and $37651.86 \mathrm{~cm}^{-1}$. Both curves were obtained by use of a slit width of $40 \mu \mathrm{m}$ in the static recording mode. The black and red curves were recorded in different pixels of the CCD by use of settings of the central pixel at absolute wavenumbers of $37695.57-700=36995.57$, and $37695.57+700=$ $38395.57 \mathrm{~cm}^{-1}$, respectively. The difference in spectral resolutions, $\Delta \omega$, is considerable, as indicated. 
As discussed in connection with Fig. 4, the resolution will depend on whether spectra measured with a multi-channel Czerny-Turner spectrometer are recorded in static or extended mode (at least for narrow bands). This feature of the dispersive CCD spectrometer systems will cause testing with the same single line to give different spectral resolution results depending on the grating angle setting. As an example we show in Fig. 8 spectra for the $265.2042 \mathrm{~nm}$ mercury line, recorded for two different central wavenumber settings. An obvious difference is clearly seen.

\section{DISCUSSION}

According to the expression of the spectral resolution (Eq. 12), the spectral resolution depends strongly (quadratic) on the absolute wavenumber of the Raman scattered light. In DUV the wavenumbers are about twice the values in the visible range. Therefore, in order to get similar spectral resolutions for shorter ultraviolet exciting wavelengths, especially in the extreme DUV, one must choose high-groove-density gratings and narrow entrance slit widths to maintain/obtain high resolution. The wavenumber position of a Raman band also has a significant effect on the spectral resolution. Figure 4 shows this trend of the spectral resolution along the Raman shift axis.

Although Eqs. 12, 13, and 14 reveal the spectral resolution in dependence of all the principal factors, the effect of diffractions and aberrations has not been determined in detail by the present theoretical approximation method. If one would examine these effects it requires information on the actual situation of the optical components and deep optical investigations. Instead of this, however, we have introduced a new approximate procedure to handle the situation, by introducing the diffractions and aberrations compensating factor $A$. This $D A C F$ factor, in Eq. 14, is used to develop a hyperbola function model for the spectral resolution with respect to the width of the entrance slits and for the different settings of the experimental situation. The experimental data obtained during this work have shown that this procedure is well suited to fit the results, as seen in Fig. 3 and checked in Fig. 7.

\section{CONCLUSION}

Often, in Raman spectroscopy, one wants to know what the true width of an observed Raman band is. We have here presented a generalized method to determine it: first one must determine the particular spectral resolution of the applied instrument at the precise position where the Raman band is located. We have demonstrated that this can been done by using Eqs. 12, 13, and 14. All the parameters except a new one, $A$ (or DACF), can be easily found, and $A$ can be determined by measuring on a given narrow line, in casu the diamond Raman fundamental at $1332.4 \mathrm{~cm}^{-1}$ or low pressure emission plasma lines from mercury gas. Then, as soon as the spectral resolution at the position of the Raman band in question is known, the band width can be found by means of Eq. 16, as derived in Appendix 1. In order to ensure the measurement accuracy, it should be noted that the grating must be turned to such an angle that the tested Raman band is located at the central part of the CCD detector, because only here is the exact condition for using Eq. 12 strictly fulfilled. Otherwise, there will be deviations as shown in Fig. 4 (black lines). One takes advantage of the feature that the central area of the CCD is in the optimized position for the slit imaging. Using the central area of the CCD is also the condition for which $A$ can be best approximated as a constant.

It is to be noted that many CCD-based Raman spectrometric systems apply so-called static recording modes, i.e., spectrometric detection of photons in many pixels of the detector while keeping the grating still (static). Instead of this, the Renishaw system has a so-called extended scan mode, in which the grating is synchronously moved coupled to the charges in the CCD pixels. ${ }^{19-23} \mathrm{We}$ conclude from our analysis here that the static and the extended recording modes differ with respect to their resolution properties as described. This dependence has been widely overlooked in the literature but can now be accounted for by use of the new method (only needed for narrow bands).

\section{ACKNOWLEDGMENTS}

The Danish Agency for Science, Technology and Innovation provided a grant (\#09-065038/FTP) for C. L. and for the UV-Raman instrumentation. DTU also contributed to make this work possible.

1. S.A. Asher, C.R. Johnson. "Raman spectroscopy of a coal liquid shows that fluorescence interference is minimized with ultraviolet excitation". Science. 1984. New Series 225. No. 4659: 311-313.

2. S.A. Asher. "Ultraviolet Raman Spectrometry". In: J.M. Chalmers, P.R. Griffiths, editors. Handbook of Vibrational Spectroscopy. New York: John Wiley and Sons Ltd., 2002. Vol. 1, Pp. 557-571.

3. D.D. Tuschel, A.V. Mikhonin, B.E. Lemoff, S.A. Asher. "Deep Ultraviolet Resonance Raman Excitation Enables Explosives Detection". Appl. Spectrosc. 2010. 64(4): 425-432.

4. A.J. Kunov-Kruse, S.B. Kristensen, C. Liu, R.W. Berg. "Experimental and theoretical Raman spectrum of the dye Sudan I". J. Raman Spectrosc. 2011. 42: 1470-1478.

5. A.C. Ferrari, J.C. Meyer, V. Scardaci, C. Casiraghi, M. Lazzeri, F. Mauri, S. Piscanec, D. Jiang, K.S. Novoselov, S. Roth, A.K. Geim. "Raman Spectrum of Graphene and Graphene Layers". Phys. Rev. Lett. 2006. 97(18): 187401-187404.

6. H. Ou, Y. Ou, C. Liu, R.W. Berg, K. Rottwitt. "Formation and Characterization of varied size germanium nanocrystals by Electron Microscopy, Raman spectroscopy and Photoluminescence". Opt. Mater. Exp. 2011. 1(4): 643-651.

7. J.M. Lerner, A. Thevenon. "The Optics of Spectroscopy, a Tutorial V2.0". Jobin-Yvon ISA Optical Systems Instruments S-A, Inc. 1988. Pp. 22.

8. C. Palmer, E. Loewen. "Diffraction Grating Handbook". Rochester, NY: Newport Corp, 2005. $6^{\text {th }}$ edition. [http://gratings.newport.com/library/ handbook/handbook.pdf.]

9. I.K. Lednev, V.V. Ermolenkov, W. He, M. Xu. "Deep-UV Raman spectrometer tunable between 193 and $205 \mathrm{~nm}$ for structural characterization of proteins". Anal. Bioanal. Chem. 2005. 381: 431-437.

10. B.T. Bowie, P.R. Griffiths. "Determination of the Resolution of a Multichannel Raman Spectrometer Using Fourier Transform Raman Spectra”. Appl. Spectrosc. 2003. 57(2): 190-196.

11. S. Bykov, I. Lednev, A. Ianoul, A. Mikhonin, A. Munro, S.A. Asher. "Steady-State and Transient Ultraviolet Resonance Raman Spectrometer for the 193-270 nm Spectral Region”. Appl. Spectrosc. 2005. 59: 1541-1552.

12. J.D. Ingle, S.R. Crouch. "Spectrochemical Analysis". Prentice Hall, Englewood Cliffs, N.J.: 1988. Pp. 70-74.

13. R.L. McCreery. "Dispersive Raman Spectrometers". In Raman Spectroscopy for Chemical Analysis. New York: Wiley Interscience, 2000. Chap. 8.

14. N. Sheppard. "The Historical Development of Experimental Techniques in Vibrational Spectroscopy". In: J.M. Chalmers, P.R. Griffiths, editors. Handbook of Vibrational Spectroscopy. Chichester: John Wiley and Sons Ltd., 2002. 1, Chpt. 1, Pp. 17-30.

15. E. Hecht. "Optics". San Francisco, CA: Addison-Wesley, 2002. Pp. 461, Pp. 468, Pp. 470. $4^{\text {th }}$ edition. ISBN: 0-321-18878-0.

16. P.R. Griffiths. "Resolution and Instrument Line Shape Function". In: J.M. Chalmers, P.R. Griffiths, editors. Handbook of Vibrational Spectroscopy. Chichester: John Wiley and Sons Ltd., 2002. Vol. 1, Chap. 2, Pp. 2-8.

17. V. Deckert, W. Kiefer. "Scanning Multichannel Technique for Improved Spectrochemical Measurements with a CCD Camera and its Application to Raman Spectroscopy". Appl. Spectrosc. 1992. 46(2): 322-328.

18. S. Prawer, R.J. Nemanich. "Raman Spectroscopy of Diamond and Doped Diamond”. Math. Phys. Eng. Sci. 2004. 362: 2537-2565. 
19. Renishaw. Technology note L-8012-3890-01-F SPD074TN, "Renishaw's SynchroScan: Artefact-free spectra". Gloucestershire, UK: Renishaw, plc., 2007. August. Pp. 2.

20. C. Dyer, B.J.E. Smith. "Application of continuous extended scanning techniques to the simultaneous detection of Raman scattering and photoluminescence from calcium disilicates using visible and near-infrared excitation”. J. Raman Spectrosc. 1995. 26: 777-783.

21. G.D. Pitt, D.N. Batchelder, R. Bennett, R.W. Bormett, I.P. Hayward, B.J.E. Smith, K.P.J. Williams, Y.Y. Yang, K.J. Baldwin, S. Webster. "Engineering Aspects and Applications of the new Raman Instrumentation". IEE Proc. Sci. Meas. Technol. 2005. 152(6): 241-318. doi:10.1049/ ip-smt:20050015.

22. D.N. Batchelder, B. Smith, C. Cheng, R.J. Chaney. Assignee: Renishaw. Spectroscopic apparatus and methods. EPO Patent 0638788 A1. Filed 1992. Issued 1995.

23. D.N. Batchelder, B. Smith, C. Cheng, R.J. Chaney. Assignee: Renishaw. Spectroscopic apparatus and methods. US Patent 5,689,333. Filed 1995. Issued 1997.

24. F.M. Phelps III, "M.I.T. "Wavelength Tables, Wavelengths by Element". The MIT Press, 1982. Cambridge, MA: Vol. 2, Pp. 274, based on data by L. Cardaun, Zeits. f. wiss. Phot. 1914. 14: 56-89.

25. V. Kaufman, B. Edlén. "Reference Wavelengths from Atomic Spectra in the Range $15 \AA$ to $25000 \AA$, J. Phys. Chem. Ref. Data. 1974. 3(4): 825-880.

\section{APPENDIX 1}

Relation Between Spectral Resolution and True Raman Bandwidth. A true Raman spectrum is here defined as a Raman spectrum excited by a hypothetically monochromatic light source, which could be a narrow section (a delta function at $\left.\omega_{1}\right)$ of the laser spectrum, $g_{\mathrm{L}}(\omega)$, as shown in Fig. A1, panel a. The profile of a true Raman band with a FWHM bandwidth of $\Delta \omega_{\mathrm{R}}$ can be described as $g_{\mathrm{R}}\left(\omega_{\mathrm{r}}\right)$ as shown in Fig. A1, panel b. Therefore, the Raman spectrum, denoted $g_{\mathrm{LR}}(\omega)$, excited by the laser, ideally should be created as a convolution result of $g_{\mathrm{L}}(\omega)$ and $g_{\mathrm{R}}\left(\omega_{\mathrm{r}}\right)$ as shown in Fig. A1, panel c.

In the next step, the real Raman spectrum $g_{\mathrm{LR}}(\omega)$ will here be denoted the spectrum of the light as it really is before the light enters into the spectrometer, i.e., not the measured spectrum. Owing to the limited spectral resolution of the spectrometer, the measured Raman signal could be considered as coming from a series of hypothetical monochromatic light increments that go through the measuring process. This implies that each element in the $g_{\mathrm{LR}}(\omega)$ spectrum would be broadened to contribute a profile of $g_{\mathrm{S}}\left(\omega_{\mathrm{S}}\right)$, shown in Fig. A1, panel d, to the measured spectrum.

By summing the contributions from all these intervals, the measured Raman spectrum, $g_{\mathrm{M}}(\omega)$, is the convolution result of $g_{\mathrm{LR}}(\omega)$ and $g_{\mathrm{S}}\left(\omega_{\mathrm{s}}\right)$, as shown in Fig. A1, panel e:

$$
g_{\mathrm{M}}(\omega)=g_{\mathrm{LR}}(\omega) \otimes g_{\mathrm{S}}\left(\omega_{\mathrm{S}}\right)=g_{\mathrm{L}}(\omega) \otimes g_{\mathrm{R}}\left(\omega_{\mathrm{r}}\right) \otimes g_{\mathrm{S}}\left(\omega_{\mathrm{S}}\right)
$$

By assuming a Gaussian approximation, the measured bandwidth of the observed Raman band is given by

$$
\Delta \omega_{\mathrm{M}}=\sqrt{\left(\Delta \omega_{\mathrm{R}}\right)^{2}+\left(\Delta \omega_{\mathrm{S}}\right)^{2}+\left(\Delta \omega_{\mathrm{L}}\right)^{2}}
$$

\section{APPENDIX 2}

Expression of Spectral Resolution for Static Grating Acquisition. When the grating is static, the angle of $\theta_{\mathrm{i}}$ is kept constant during the measurement of the Raman photons (see Fig. 1). The Raman wavenumber shift of signal incident on the center of the CCD is denoted as $\omega_{0}$ (not shown in Fig. 1).

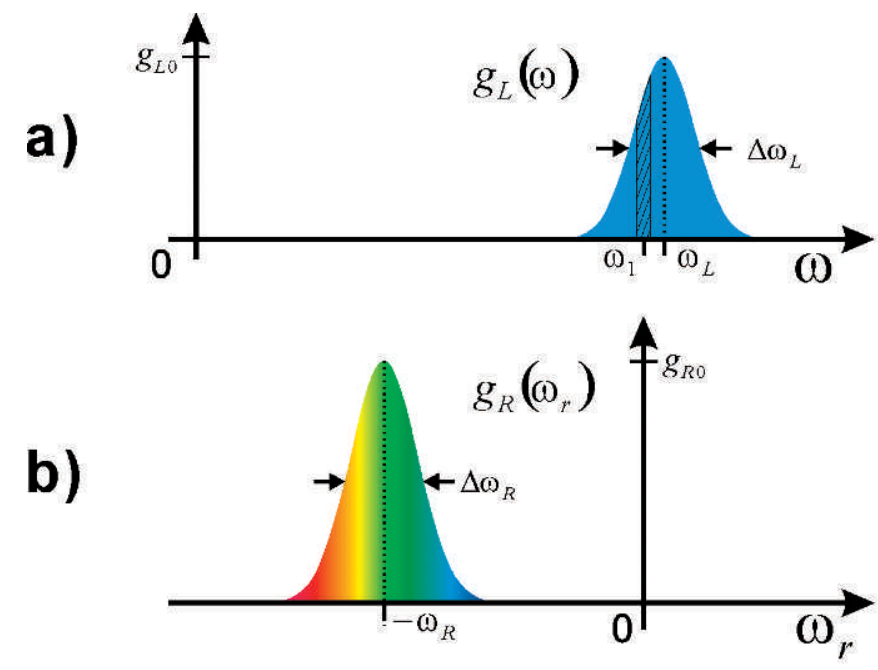

c)

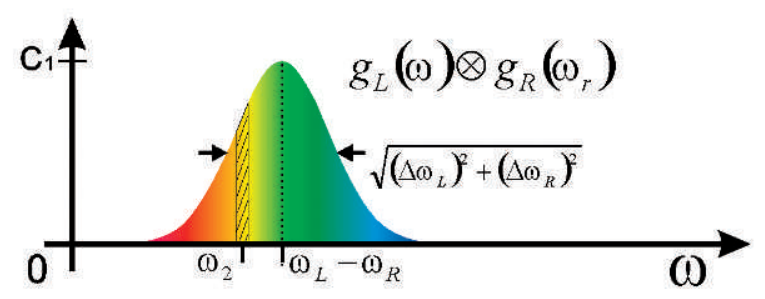

d)

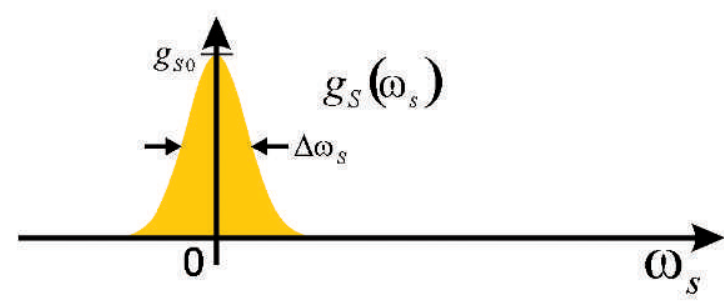

e)

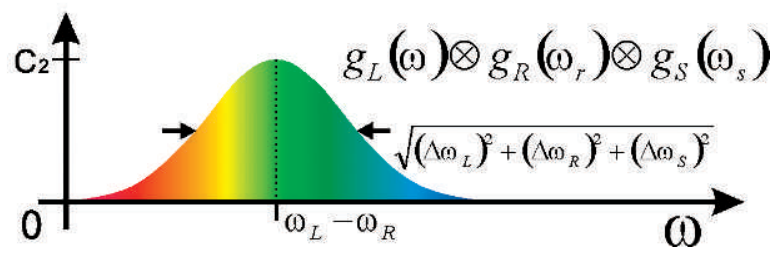

FIG. A1. The relationship between the laser spectrum, the true Raman spectrum, the real Raman spectrum, the spectral resolution of spectrometer, and the measurement of the Raman spectrum under a Gaussian profile approximation: (a) the laser spectrum with a monochromatic incremental section $\omega_{1}$; (b) the true Raman spectrum; (c) the real Raman spectrum; (d) a hypothetical monochromatic signal broadened by the spectral resolution of the spectrometer; and (e) the measurement of the Raman band.

Therefore, equation Eq. 8 can be modified as

$$
\theta=\arcsin \left[\frac{\frac{1}{\left(\omega_{\mathrm{L}}-\omega_{0}\right)}}{2 \cdot d \cdot \cos \left(\frac{\alpha}{2}\right)}\right]+\frac{\alpha}{2}
$$

The constant $\theta_{\mathrm{i}}$ can be found by substituting Eq. A2.1 into Eq. 5 to get:

$$
\theta_{i}=\arcsin \left[\frac{\frac{1}{\left(\omega_{\mathrm{L}}-\omega_{0}\right)}}{2 \cdot d \cdot \cos \left(\frac{\alpha}{2}\right)}\right]-\frac{\alpha}{2}
$$


By substituting Eq. A2.2 into Eq. 1 we get

$$
\theta=\arcsin \left(\frac{\lambda}{d}-\sin \left\{\arcsin \left[\frac{\frac{1}{\left(\omega_{\mathrm{L}}-\omega_{0}\right)}}{2 \cdot d \cdot \cos \left(\frac{\alpha}{2}\right)}\right]-\frac{\alpha}{2}\right\}\right)
$$

Here it should be noted that $\theta$ in Eq. A2.3 is a general expression and not only the condition corresponding to the center of the CCD. Furthermore $\theta$ is a function of $\lambda$.

If we substitute Eq. A2.3 into Eq. 4 and use unit conversion and Eqs. 10 and 11, we get:

$$
\begin{aligned}
& \Delta \omega\left[\omega_{\mathrm{L}}, \omega_{\mathrm{R}}, G, f_{2}, \alpha, b_{\mathrm{img}}\right] \\
& \quad=\left(\omega_{\mathrm{L}}-\omega_{\mathrm{R}}\right)^{2} \cdot \frac{b_{\text {img }}}{G \cdot f_{2}} \\
& \cdot \cos \left[\arcsin \left(\frac{G}{\left(\omega_{\mathrm{L}}-\omega_{\mathrm{R}}\right)}-\sin \left\{\arcsin \left[\frac{G}{2\left(\omega_{\mathrm{L}}-\omega_{0}\right) \cdot \cos \left(\frac{\alpha}{2}\right)}\right]-\frac{\alpha}{2}\right\}\right)\right]
\end{aligned}
$$

In Fig. A2, we show black lines for the resolution versus the Raman shift, calculated from the above equation for different values of the static setting $\omega_{0}$ of the grating. The black lines show an unusual (artifact) behavior: the spectral resolution can reach 0 . Actually, in this case, the grating diffraction angle $\theta$ is simultaneously approaching $90^{\circ}$. Such an angle, see Fig. 1, is

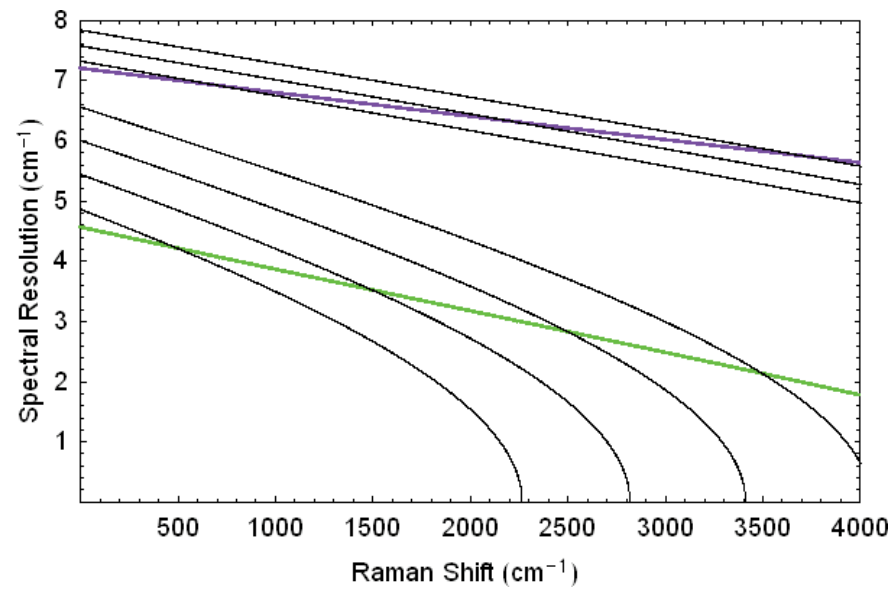

FIG. A2. Spectral resolution along the Raman shift axis. The green and violet lines show the theoretical results calculated by use of Eq. 12 for the central part of the CCD (in extended scanning mode using only a few pixels). The black lines show the spectral resolution in four and three spectrograms excited with 514.5 and $257.3 \mathrm{~nm}$ lasers, respectively, recording in static mode, using many pixels and settings of the central pixel of the CCD at Raman shift wavenumbers of $\omega_{0}$ for $514.5 \mathrm{~nm}=500,1500,2500$, and $3500 \mathrm{~cm}^{-1}$, and $\omega_{0}$ for $257.3 \mathrm{~nm}=$ 750,2250 , and $3750 \mathrm{~cm}^{-1}$.

not a condition that can be realized because the grating will be required to have an infinite area in order to be able to reflect the light. 